\title{
Evaluation of the peripheral dose and the conformity index for three stereotactic radiotherapy techniques: Arcs, noncoplanar fixed fields and intensity modulation
}

\author{
Hany Ammar1, Eman Eldebawy1,2, Ehab Maarouf ${ }^{1,2}$, Wafaa Khalil ${ }^{3}$, MS Zaghloul1,2 \\ ${ }^{1}$ Department of Radiotherapy, Children's Cancer Hospital Egypt \\ ${ }^{2}$ Department of Radiotherapy and Nuclear Medicine, National Cancer Institute, Cairo University \\ ${ }^{3}$ Department of Biophysics, Faculty of Science, Cairo University
}

Received April 28, 2014; Revised July 03, 2014; Accepted July 04, 2014; Published Online August 23, 2014

\section{Original Article}

\begin{abstract}
Purpose: The main aim of this work is to compare the conformity index and the peripheral doses absorbed during stereotactic treatment of a brain lesion delivered using three stereotactic techniques; Arcs, noncoplanar fixed conformal fields (3DCRT) and intensity modulation (IMRT). Methods: Ten patients with brain lesions who were previously treated with Stereotaxy radiosurgery (SRS) using the cones (Arcs technique), were re-planned both non-coplanar 3D Conformal fixed fields (3DCRT) and non-coplanar intensity modulated radiotherapy fixed fields (IMRT) with the same arrangement and orientation of the fields as the Arcs technique. To facilitate direct comparison between the competing techniques, the primary planning object was to cover $95 \%$ of the volume of PTV by $95 \%$ of the prescribed dose (1200 cGy). Results: The IMRT technique shows the highest dose conformity, Arcs technique is the next homogeneity followed by 3DCRT. The differences were statistically significant among the three different techniques $(\mathrm{P}<0.01)$ for PITV (defined as the ratio of the prescription isodose volume (PI) and Target Volume (TV)) and Conformal Number (CN). The mean integral dose using the 3 different techniques for all studied patients were: $2.44 \pm 0.36 \mathrm{~J}, 2.31 \pm 0.37 \mathrm{~J}$ and $2.6 \pm 0.37 \mathrm{~J}$ in the Arcs, IMRT and 3DCRT techniques respectively, the differences were not statistically significant $(\mathrm{p}=0.326)$. The results show that Arcs technique has the lowest volume of the body that received $2 \mathrm{~Gy}$, IMRT is the next followed by 3DCRT and IMRT technique has the lowest volume of the body that received 5 Gy, Arcs technique is the next followed by 3DCRT. The differences were not statistically significant $\mathrm{p}=0.126$ for V2 and $\mathrm{p}=0.118$ for V5, but these differences might be clinically significant that need more clinical discussion and investigation. IMRT plan delivery time almost has two folded more than the two others techniques, and the arc the technique has the lowest estimated time compared to both IMRT and Conformal techniques. The differences were statistically significant $(\mathrm{P}<0.01)$. Conclusion: The conformity index, dose homogeneity and the outfield dose are important aspects of plan quality, although they do not always receive clinically attention. Our results have been showed that the superiority of the IMRT in conformity, dose homogeneity and the lowest volume that received $5 \mathrm{~Gy}$, Arcs technique has the superiority in lower treatment delivery time than IMRT and the lowest volume that received $2 \mathrm{~Gy}$. The 3DCRT don't present a significant advantage among the competing techniques. Oncologist should be alert of the possibility of significantly increasing the secondary cancer risk particularly for pediatric patients, because children are more susceptible to the risk of second cancers.
\end{abstract}

Keywords: Radiosurgery; Conformity Index; Integral Dose; Peripheral Dose

\section{Introduction}

Linear Accelerator (Linac) arc based stereotactic radiotherapy is used with increasing frequency to treat brain tumors. This approach can be used for single (stereotactic radiosurgery (SRS)), or fractionated treatments (stereotactic radiotherapy (SRT)), It is typically carried out with circular collimators which are optimal for small, spherical targets. Treatment planning using fixed non-coplanar beams or intensity-modulated beams may enhance the ability to conform to irregularly shaped and/or large tumors, especially when combined with stereotactic localization. ${ }^{1}$ Despite the highly localized doses that may be delivered via stereotactic radiation, a small dose is nonetheless delivered to out of field region, which may cause detriment to the patient. Peripheral dose (PD) raises questions of treatment optimization, particularly in cases where patients have a long life expectancy in which secondary effects may become clinically manifest, 
especially in the treatment of pediatric patients or in those with a non-malignant primary. ${ }^{2}$

Peripheral dose results from leakage through the collimator/source head, from scattered radiation within the patient and from surrounding materials. Knowledge of peripheral dose is essential in evaluation of radiation protection impact to patient during the treatment. However, determination of the peripheral doses, such as the dose to critical organs of patients has been the subject of extensive investigation. The values were derived from measurements of contributions to the PD, i.e., from radiation scattered in the patient, leakage radiation and radiation scattered from the collimator/source. The choices of treatment machine and specific peripheral dose data are quite useful in risk reduction and, perhaps, in the selection of optimal treatment technique. ${ }^{3}$

One goal of radiosurgery is to design a treatment plan in which the prescription isodose line covers the target with a minimal excess volume and a sharp dose. Several different conformity indices have been reported to describe the conformity of the prescription isodose to the target volume. The PITV recommended to be determined by the Radiation Therapy Oncology Group RTOG. The PITV is defined as the ratio of the prescription isodose volume (PI) to the target volume (TV). Conformity indices are used to compare competing plans, evaluate treatment technique, and assess clinical complications by quantifying the dose conformity to a target volume. Several different indices have been reported to evaluate the conformity of prescription isodose surface to the target volume. ${ }^{4}$

The primary beam and its immediate periphery (in-field), as well as in regions far from the primary field (out-of field), Poor knowledge of in-field characteristics may lead to reduced treatment efficacy, whether by under-dosage of targeted tumours or over-irradiation of adjacent healthy tissues. Poor knowledge of out-of-field characteristics may result in adverse health consequences, such as radiation-induced carcinogenesis. The increasing success of radiotherapy is resulting in longer patient lifetimes, and as such there is greater time in which (typically latent) radiation-induced cancers may become manifest. Peripheral dose raises questions of treatment optimization, particularly in cases where patients have a long life expectancy in which secondary effects may become manifest, such as in the treatment of pediatric patients or those with a non-malignant primary. ${ }^{5}$

The main components of peripheral dose are leakage from LINAC head, scattered radiation from collimators and leaves, and scattered radiation within the patient. The first two contributors depend on the technique employed, as well as the device and its layout. Scattered radiation within the patient, in turn, depends on radiation energy, the distance from the edges of the field, and, to a lesser extent, the field size. ${ }^{6}$ Secondary tumour induction may seem a relatively signifi- cant problem, since the patient has to survive the primary tumour long enough for a secondary one to becomeobvious. ${ }^{7}$

A goal of stereotactic radiosurgery (SRS) is to achieve optimal dose-volume conformity (i.e., to limit the prescription dose to a volume closely coincident with the target volume), thus excluding normal tissue (NT) from the high-dose region. It has been shown that complications from SRS are related to the volume of NT receiving a minimal dose. Conversely, it is important to maintain a high degree of coverage of the target volume (TV) by the prescription dose, because a reduction in dose to spare normal structures has been shown to lead to an unacceptable rate of tumor recurrence. ${ }^{8} \mathrm{~A}$ knowledge of the doses to be deposited outside the treatment volume beforehand may allow a risk estimate of detrimental effects and, if possible, precautions to minimize the probability of developing them. To this end, several studies have been carried out with the aim of identifying different components of peripheral dose and the possible actions that can be taken to minimize the risk associated with these peripheral doses.

\section{Methods and Materials}

\section{Patients}

Ten patients with different tumor sizes, shapes and localizations were selected for this study. All selected patients were treated with stereotactic radiosurgery (SRS) at Children's Cancer Hospital, Egypt, (CCHE). The target volumes ranged from 6 to 16 CC. Three plans were performed created for each patient. These treatment plans were generated using three different techniques: Arcs, noncoplanar fixed fields and intensity modulation techniques. Each plan will individually be optimized according to the selected treatment techniques. Beam angles were chosen to minimize doses to the critical structures and to achieve high dose fall-off around the target at the same time. A Linear Accelerator $6 \mathrm{MV}$ arc based stereotactic (Siemens ONCOR ${ }^{\mathrm{TM}}$ Impression Linear Accelerator). Attached to Circular Collimators (Radionic, Integra Radionics, Burlington, MA) have maximum cone of $40 \mathrm{~mm}$ diameter or Siemens Moduleaf Collimator (mMLC; leaf projection at the isocenter: $4 \mathrm{~mm}$ ) has weight $39.7 \mathrm{Kg}$ with Cosmic 2.3 software.

Ten patients with brain lesions who were previously treated with Stereotaxy radiosurgery (SRS) using the cones (Arcs technique), were re-planned both non-coplanar 3D Conformal fixed fields (3DCRT) and non-coplanar intensity modulated radiotherapy fixed fields (IMRT) with the same arrangement and orientation of the fields as the Arcs technique. The selected cases were chosen to represent varying tumor shape, size, location and proximity to critical organs. The mean planning target volume (PTV) of the selected cases was $10.9 \pm 3.7 \mathrm{~cm}^{3}$ (ranged $\left.6 \mathrm{~cm}^{3}-16 \mathrm{~cm}^{3}\right)$. 
To maintain the accuracy and precision desired in SRS two basic conditions were used: immobilization of the patient during the treatment and the exact determination of tumor position. These two conditions were promptly achieved using an invasive frame and localizer.

A physician screws the stereotactic frame on the patient's head. Using of frame solves two problems: 1) minimize the chance of head movement with respect to frame (immobilization) and 2) it sets up an external frame with respect to which co-ordinates of the tumor could be defined (localization). To determine the co-ordinate of the tumor a localization frame with $\mathrm{N}$-shape fiducial rods is attached to the head frame before the patient undergoes imaging.

Diagnostic imaging modalities used in stereotactic radiosurgery are 1) computed tomography (CT) and 2) Magnetic Resonance Imaging (MRI).

By using an advanced software (radionics software), it is possible to superimpose geometrically CT and MRI images to use the information of both image modalities. This process is called "fusion". Once the images are fused, different viewing tools allow the planner to view the images superimposed on top of each other. The goal of SRS planning is to deliver the maximum dose to the tumor and the minimum dose to the surrounding healthy tissues. Each patient was scanned by CT while fixed in the stereotactic head frame and localizer. Scan was made with 2-mm slice thickness. All patients also had an MRI scan of the brain.

\section{Treatment Planning}

Once the imaging is complete, data is transferred to a Treatment Planning System (TPS). In the TPS all the CT image slices are localized. The fiducials of the localizer box are found to determine the co-ordinate system used for planning, then image fusion between CT and MRI images is performed and the physician contours the target and the organ at risks (OAR) structures on the fused image slices.

Once the target and the OARs are contoured, a radiosurgery plan may be developed. Three plans will be created for each patient. Three treatment plans have been generated using three techniques: ARCs, non-coplanar (3DCRT) fixed fields and non-coplanar (IMRT), the three plans have the same orientation and arrangement of the fields. First ARC plan was made, the OARs were avoided as much as possible by selecting the optimal table positions, arc angles and the most fitted cone size, then Arc plan was converted into 3DCRT and IMRT fixed fields plans. Beam shaping for 3DCRT and IMRT were carried out using Siemens Moduleaf of $2.5 \mathrm{~mm}$ width.

Each plan has been individually optimized according to the treatment techniques selected. All plan parameters were re-chosen to minimize doses to the critical structures and to achieve high dose fall-off around the target at the same time. All treatment plans were made on Radionics treatment planning system X-Knife radionics by using primary + scatter algorithm. The adaptation of the dose-volume constraints on an individual basis enabled better avoidance of critical organs.

The primary objective for each plan was to $95 \%$ of the Planning target volume (PTV) covered by $95 \%$ of the prescribed dose while minimizing the dose to OARs. All plans were generated using a single isocenter. A dose of $12 \mathrm{~Gy}$ in a single fraction was prescribed and normalized to the isocenter.

\section{Plan Quality}

\section{Dose Volume Histogram}

All plans were optimized such that $95 \%$ of the volume of PTV received $95 \%$ of the prescribed dose of $12 \mathrm{~Gy}$.

\section{Conformity Indices}

One goal of radiosurgery is to design a treatment plan in which the prescription isodose line covers the target with a minimal excess volume and a sharp dose fall-off outside the target volume. However, planning or "mapping" the prescription isodose to a specific target shape can be a challenging task. Several different conformity indices have been reported to describe the conformity of the prescription isodose to the target volume.

The PITV recommended in the Radiation Therapy Oncology Group (RTOG) radiosurgery guidelines is probably the most frequently quoted. ${ }^{8}$ The PITV Equation 1 is defined as the ratio of the prescription isodose volume (RI) to the target volume (TV). The RTOG guidelines define a ratio of 1.0-2.0 as per protocol and ratios in the range of $0.9-1.0$ or $2.0-2.5$ as minor variations.

$$
P I T V=\frac{V_{R I}}{T V}-----(1)
$$

The PITV index presents a major drawback: It can never take into account the degree of spatial intersection of two volumes or their shapes. In extreme cases, it may be equal to 1 while these two volumes are situated away from each other and present.

Another Index was used in this study called Conformation Number (CN) ${ }^{8}$, A conformation number to quantify the degree of conformality in brachytherapy and external beam irradiation was a good illustration example. Calculation of this CN simultaneously takes into account irradiation of the target volume and irradiation of healthy tissues as shown in Equation 2

$$
C N=\frac{T V_{R I}}{T V} \times \frac{T V_{R I}}{V_{R I}}-----(2)
$$


Where:

RI: Reference isodose,

TV: Target volume,

OAR: Organ at Risk,

TVRI: Target volume covered by the reference isodose, $\mathrm{V}$ RI: Volume of the reference isodose.

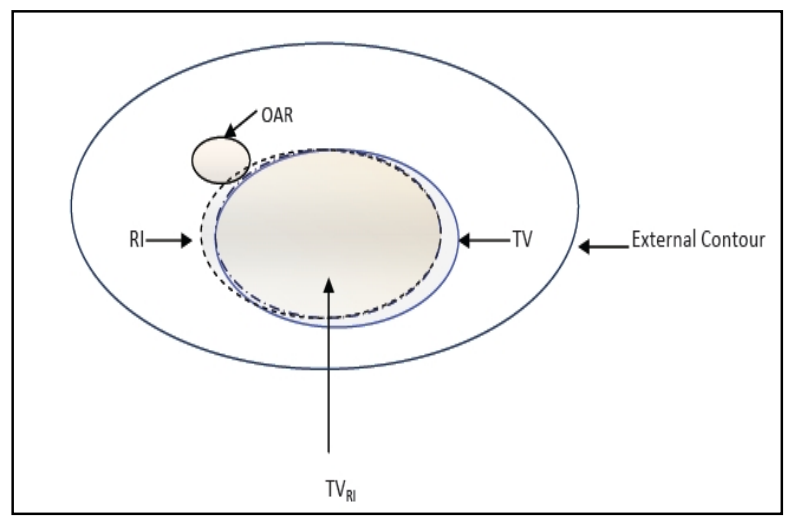

FIG. 1: Shows the various volumes required to calculate the PITV and $\mathrm{CN}$.

\section{Integral Dose}

The integral dose is reported as the sum of all dose voxel times their mass ${ }^{10}$, as shown in Equation 3.

$$
\left.\begin{array}{l}
E_{\text {integral }}=N \times D_{\text {mean }} \times m_{\text {voxel }} \\
E_{\text {integral }}=m_{\text {body }} \times D_{\text {mean }, \text { body }}
\end{array}\right\}----(3)
$$

Where, N: the number of voxels,

Dmean: The mean dose to the body contour,

mvoxel: The mass of a voxel.

Although for a proper evaluation of integral dose different density values should be considered for different structures, for the sake of simplicity a constant density $\rho=1 \mathrm{~g} / \mathrm{cm}^{3}$ was assumed for all structures.

\section{Volume receiving $2 \mathrm{~Gy}$ and $5 \mathrm{~Gy}$}

Some models of radiation carcinogenesis suggest that the dose-response relationship is linear up until a dose $6 \mathrm{~Gy}$, where it then reaches plateau. ${ }^{11}$ The volume receiving 2 Gy and $5 \mathrm{~Gy}$ were reported in this study for each treatment plan.

\section{Estimation of treatment delivery time}

Recorded of the monitor units $(\mathrm{Mu})$ of each treatment plan gave us a reasonable data to estimate the treatment delivery time.

\section{Statistics}

For the statistical analysis we used SPSS, version 20 (SPSS Inc., Chicago, IL). To analyze the differences between the Arcs, 3DCRT and IMRT plans, ANOVA test was used for the three techniques. The level of statistical significance was considered $\mathrm{p}<0.05$ for all calculation; therefore, a 95\% confidence interval was applied.

\section{Results}

\section{Dose Volume Histogram}

The dose volume evaluation for the target with the three different techniques was performed. The data for all patients is presented in Table 4.1. All Plans were optimized such that $95 \%$ of the volume of the PTV received $95 \%$ of the prescribed dose. Dose volume criteria are illustrated in Figure 4.1. Where the minimum dose is reported as the dose to the 99.5\% of the PTV and the maximum dose is reported as the dose to $0.5 \%$ of the PTV.

TABLE 1: Listed the minimum, maximum, dose homogeneity and mean dose for the competing plans of all the patients

\begin{tabular}{|c|c|c|c|c|}
\hline & $\begin{array}{c}\text { Minimum } \\
\text { Dose } \\
\text { (cGy) }\end{array}$ & $\begin{array}{c}\text { Maximum } \\
\text { Dose } \\
\text { (cGy) }\end{array}$ & $\begin{array}{c}\text { Dose } \\
\text { Inhomo } \\
\text { geneity }\end{array}$ & $\begin{array}{l}\text { Mean } \\
\text { Dose } \\
\text { (cGy) }\end{array}$ \\
\hline \multicolumn{5}{|l|}{ Patient 1(6 CC) } \\
\hline Arcs & 1112.3 & 1217.2 & 104 & 1184.1 \\
\hline IMRT & 1122.4 & 1202.7 & 80 & 1165.3 \\
\hline Conformal & 1110 & 1244.2 & 134.2 & 1203.3 \\
\hline \multicolumn{5}{|l|}{ Patient 2(7 CC) } \\
\hline Arcs & 1107.8 & 1287.7 & 179.8 & 1204.6 \\
\hline IMRT & 1105.9 & 1218.7 & 112.8 & 1166.4 \\
\hline Conformal & 1123.5 & 1331.6 & 208 & 1242.9 \\
\hline \multicolumn{5}{|l|}{ Patient 3(7.12 CC) } \\
\hline Arcs & 1116 & 1214 & 98 & 1178 \\
\hline IMRT & 1130 & 1218 & 88 & 1182 \\
\hline Conformal & 1108 & 1242 & 134 & 1196 \\
\hline \multicolumn{5}{|l|}{ Patient $4(8.47 \mathrm{CC})$} \\
\hline Arcs & 1109.5 & 1239.3 & 129.8 & 1186.4 \\
\hline IMRT & 1120.3 & 1218.8 & 98.5 & 1173.9 \\
\hline Conformal & 1103.6 & 1253.7 & 150.1 & 1190.2 \\
\hline \multicolumn{5}{|l|}{ Patient 5(10.26 CC) } \\
\hline Arcs & 1108.1 & 1279.3 & 171.2 & 1194.6 \\
\hline IMRT & 1109.8 & 1216.5 & 106.7 & 1181 \\
\hline Conformal & 1101.3 & 1294.7 & 193.4 & 1214.7 \\
\hline \multicolumn{5}{|l|}{ Patient 6(11.88 CC) } \\
\hline Arcs & 1128.4 & 1222 & 93.5 & 1184.3 \\
\hline IMRT & 1130.2 & 1216.3 & 86.1 & 1176 \\
\hline Conformal & 1120 & 1280 & 140 & 1207.3 \\
\hline \multicolumn{5}{|l|}{ Patient 7(12.12 CC) } \\
\hline Arcs & 1112.8 & 1263.3 & 150 & 1182.7 \\
\hline IMRT & 1116 & 1240 & 124 & 1178 \\
\hline Conformal & 1109.4 & 1268.7 & 159.3 & 1198 \\
\hline \multicolumn{5}{|l|}{ Patient 8(13.12 CC) } \\
\hline Arcs & 1084.9 & 1221 & 136 & 1176.6 \\
\hline IMRT & 1112.5 & 1208.9 & 96.5 & 1172.6 \\
\hline Conformal & 1075.4 & 1229.9 & 154.5 & 1183.9 \\
\hline \multicolumn{5}{|l|}{ Patient $9(14.62 \mathrm{CC})$} \\
\hline Arcs & 1121 & 1279 & 158 & 1233 \\
\hline IMRT & 1125 & 1261 & 136 & 1196.8 \\
\hline Conformal & 1105 & 1288 & 182.4 & 1225.5 \\
\hline \multicolumn{5}{|l|}{ Patient10(15.77 CC) } \\
\hline Arcs & 1160 & 1287.9 & 127.7 & 1219.2 \\
\hline IMRT & 1109.9 & 1208.2 & 98.3 & 1175.9 \\
\hline Conformal & 1149.4 & 1297.3 & 147.9 & 1261 \\
\hline
\end{tabular}




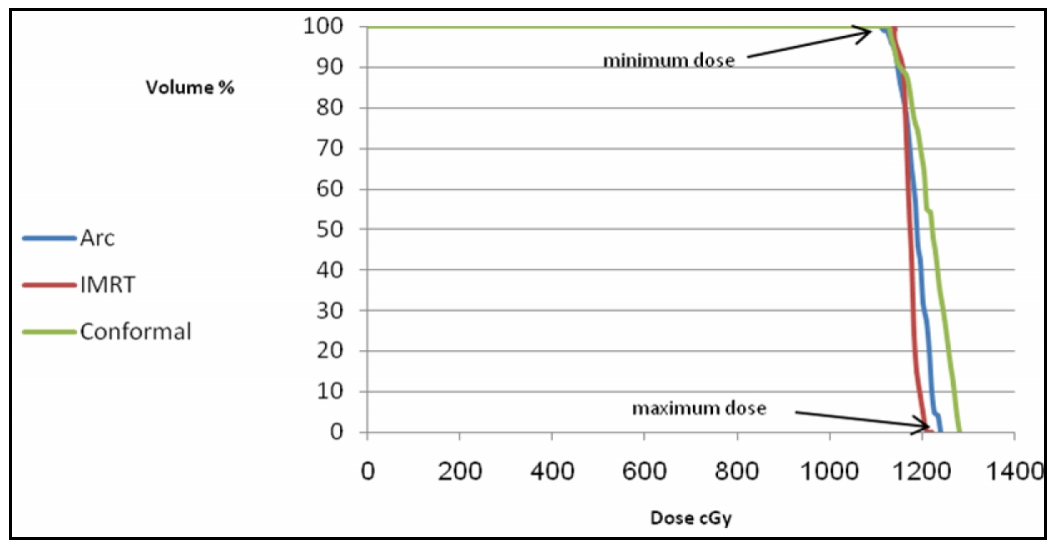

FIG. 2: Dose volume histogram criteria.

\section{Conformity Indices}

Table 2 summarizes the PITV conformity index (C.I) and the conformal number between the three different stereotactic techniques.

TABLE 2: The PITV and Conformation Number (CN) for the competing plans.

\begin{tabular}{|c|c|c|c|c|c|c|}
\hline & \multicolumn{2}{|c|}{ Arcs } & \multicolumn{2}{|c|}{ IMRT } & \multicolumn{2}{|c|}{ Conformal } \\
\hline & PITV & $\mathrm{CN}$ & PITV & $\mathrm{CN}$ & PITV & $\mathrm{CN}$ \\
\hline Patient 1(6 CC) & 1.5 & 0.6 & 1.3 & 0.81 & 1.5 & 0.6 \\
\hline Patient 2(7 CC) & 1.2 & 0.77 & 1.1 & 0.88 & 1.3 & 0.69 \\
\hline Patient 3(7.12 CC) & 1.4 & 0.65 & 1.3 & 0.77 & 1.4 & 0.65 \\
\hline Patient 4(8.47 CC) & 1.3 & 0.73 & 1.1 & 0.86 & 1.3 & 0.68 \\
\hline Patient 5(10.26 CC) & 1.4 & 0.68 & 1.2 & 0.79 & 1.5 & 0.68 \\
\hline Patient 6(11.88 CC) & 1.3 & 0.7 & 1.1 & 0.88 & 1.5 & 0.61 \\
\hline Patient 7(12.12 CC) & 1.6 & 0.69 & 1.2 & 0.77 & 1.6 & 0.69 \\
\hline Patient 8(13.12 CC) & 1.4 & 0.73 & 1.1 & 0.89 & 1.4 & 0.73 \\
\hline Patient 9(14.62 CC) & 1.5 & 0.64 & 1.3 & 0.74 & 1.5 & 0.64 \\
\hline Patien10(15.77 CC) & 1.2 & 0.72 & 1.1 & 0.87 & 1.4 & 0.64 \\
\hline Mean $\pm S D(10.6 \pm 3.4$ CC $)$ & $1.4 \pm 0.13$ & $0.7 \pm 0.05$ & $1.2 \pm 0.09$ & $0.83 \pm 0.05$ & $1.4 \pm 0.09$ & $0.67 \pm 0.04$ \\
\hline \multicolumn{3}{|c|}{ PITV comparisons } & & \multicolumn{3}{|c|}{ CN comparisons } \\
\hline \multicolumn{3}{|c|}{$\begin{array}{l}\text { P Value for comparison between three groups } \\
\text { (ANOVA): }<0.01 \\
\text { Pairwise comparison: } \\
\text { IMRT vs. Arcs: }<0.003 \\
\text { IMRT vs. Conformal: }<0.01 \\
\text { Arcs vs. Conformal: } 0.598\end{array}$} & & \multicolumn{3}{|c|}{$\begin{array}{l}\text { P Value for comparison between three groups } \\
\text { (ANOVA): }<0.01 \\
\text { Pairwise comparison: } \\
\text { IMRT vs. Arcs: }<0.01 \\
\text { IMRT vs. Conformal: }<0.01 \\
\text { Arcs vs. Conformal: } 0.405\end{array}$} \\
\hline
\end{tabular}

The chart bars (Figure 3 and Figure 4) represent the PITV conformity index and the Conformal Number, respectively.

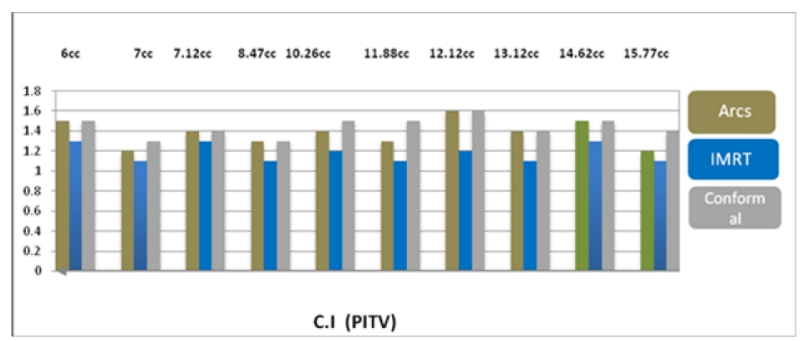

FIG. 3: PITV conformity index for the three different Stereotactic Techniques.

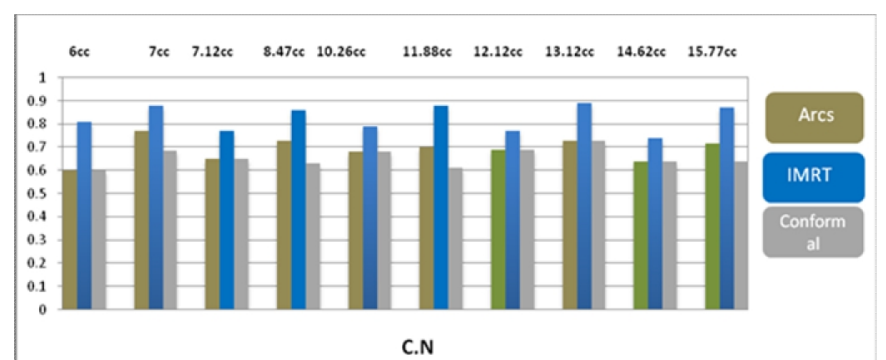

FIG. 4: Conformal Number for the three different Stereotactic Techniques 


\section{Integral Dose}

The mean integral dose using the 3 different techniques for all studied patients were: $2.44 \pm 0.36,2.31 \pm 0.37$ and $2.6 \pm$ 0.37 in the Arcs, IMRT and 3DCRT techniques respectively.

The differences were not statistically significant $(\mathrm{p}=0.326)$ (Table 3 and Figure 5).

TABLE 3: The integral dose in joule for the three different stereotactic techniques.

\begin{tabular}{lccc}
\hline \hline & \multicolumn{3}{c}{ Integral Dose } \\
\hline & Arcs & IMRT & Conformal \\
\hline Patient 1(6 CC) & 1.89 & 1.8 & 1.98 \\
Patient 2(7 CC) & 2.16 & 1.99 & 2.31 \\
Patient 3(7.12 CC) & 2.19 & 2.1 & 2.34 \\
Patient 4(8.47 CC) & 2.24 & 2.13 & 2.4 \\
Patient 5(10.26 CC) & 2.33 & 2.18 & 2.47 \\
Patient 6(11.88 CC) & 2.4 & 2.2 & 2.5 \\
Patient 7(12.12 CC) & 2.53 & 2.42 & 2.62 \\
Patient 8(13.12 CC) & 2.62 & 2.51 & 2.77 \\
Patient 9(14.62 CC) & 2.89 & 2.8 & 2.93 \\
Patien10(15.77 CC) & 3.1 & 2.99 & 3.3 \\
\hline Mean \pm SD(10.6 3.4 CC) & $2.44 \pm 0.36$ & $2.31 \pm 0.37$ & $2.6 \pm 0.37$ \\
P Value (ANOVA) & & 0.326 & \\
\hline \hline
\end{tabular}

The Chart bars Figure 5 represents the integral dose in joule.

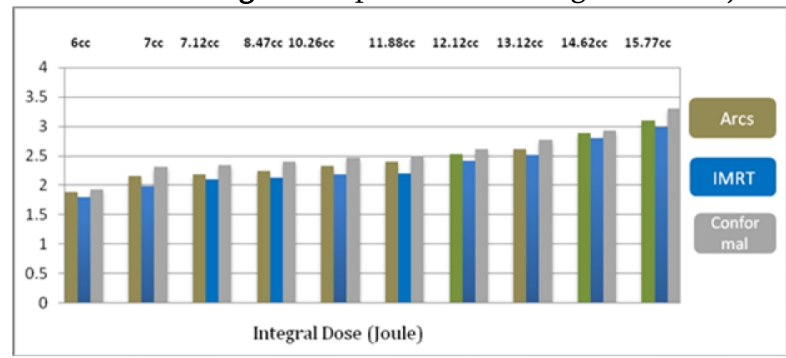

FIG. 5: The Integral dose for the three different techniques.

The integral dose is defined as (the energy deposited in the unit mass of the body). In the present work the integral dose was calculated to be equal of the mean dose in Gy for the all body and the mass of the body in kilograms $(\mathrm{kg})$. For simplicity, the mass of the body was taken as the product of its volume and a tissue density of $1 \mathrm{~g} / \mathrm{cm}^{3}$. Based on the meaning the integral dose a specific approach has been introduced that a isodose line in Gy for $1 \mathrm{~kg}$ of the body could be represent the integral dose as shown in Figures 6 and 7.

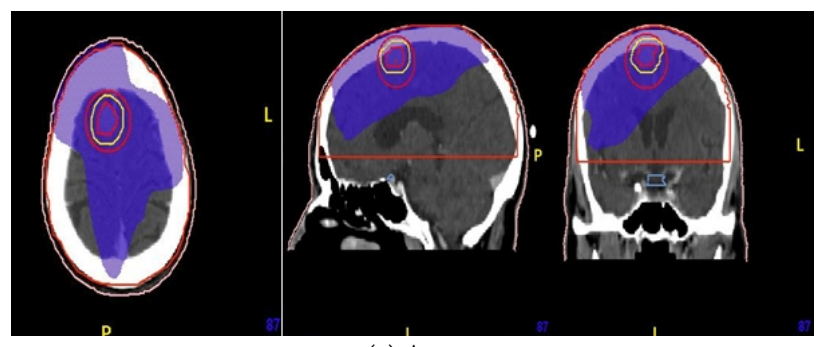

(a) Arcs

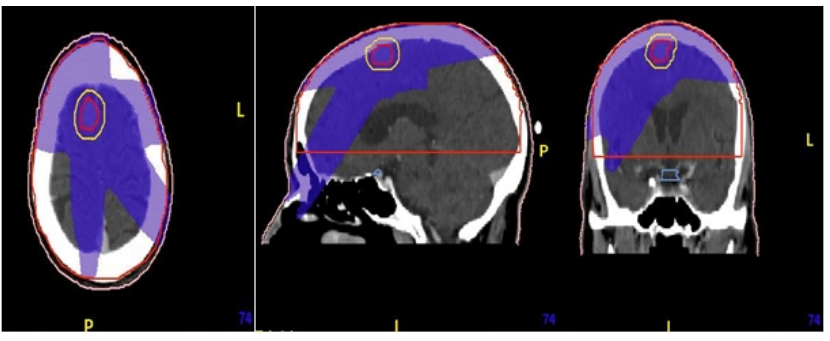

(b)IMRT

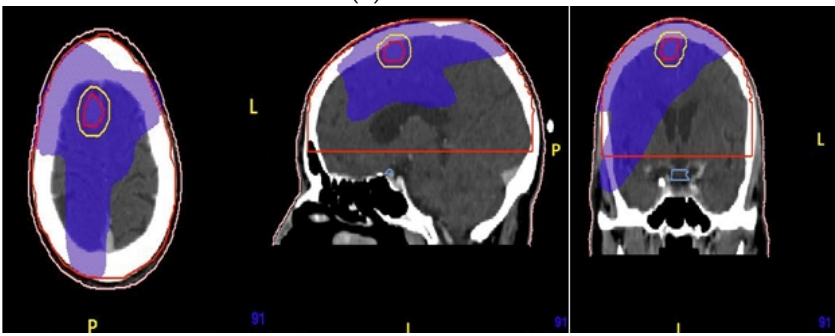

(c) 3DCRT

FIG. 6: The integral dose in axial, sagittal and coronal views of 7.12 CC PTV for three different techniques (a) Arcs, (b) IMRT, and (c) 3DCRT.

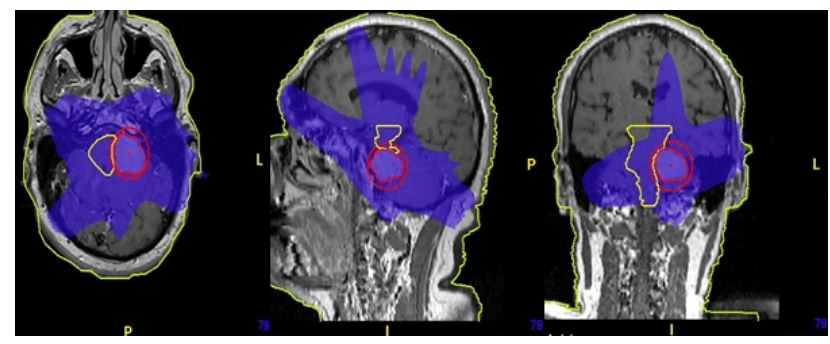

(a) Arcs

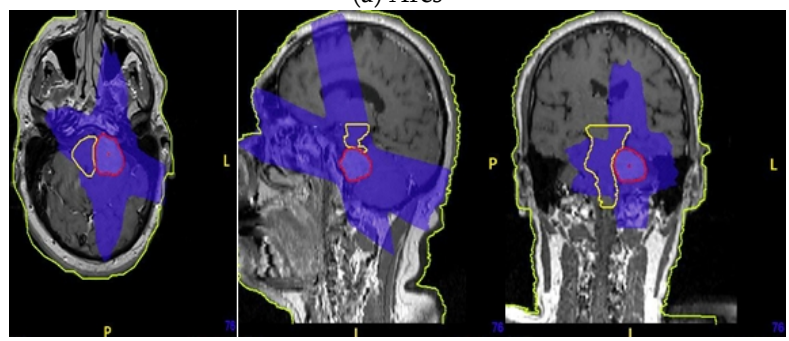

(b)IMRT

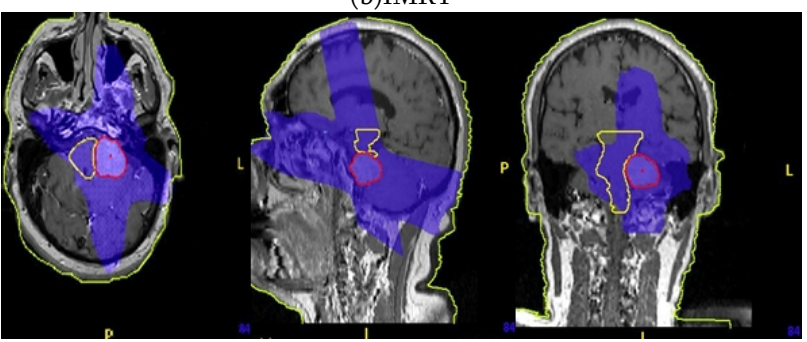

(c) 3DCRT

FIG. 7: The integral dose in axial, sagittal and coronal views of 13.12 CC for three different techniques (a) Arcs, (b) IMRT, and (c)3DCRT.

Volume receiving $2 \mathrm{~Gy}$ and $5 \mathrm{~Gy}$

The volumes that received 2 Gy and 5 Gy have been calculated for all patients where the body contour of each patient has been delineated and became represented in the DVH as shown in Figure 8. 


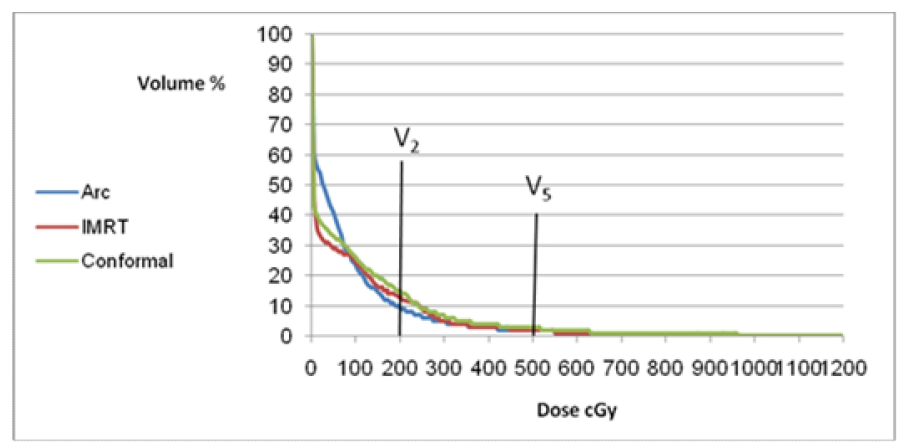

FIG. 8: Derivation of 2Gy and 5Gy by using the DVH.

TABLE 4: Summarizes the volumes in $\mathrm{cm}^{3}$ that receive $2 \mathrm{~Gy}\left(\mathrm{~V}_{2}\right)$ and $5 \mathrm{~Gy}\left(\mathrm{~V}_{5}\right)$ for the three different stereotactic techniques.

\begin{tabular}{|c|c|c|c|c|c|c|}
\hline & \multicolumn{2}{|c|}{ Arcs } & \multicolumn{2}{|c|}{ IMRT } & \multicolumn{2}{|c|}{ Conformal } \\
\hline & $\mathrm{V}_{2}$ & $\mathrm{~V}_{5}$ & $\mathrm{~V}_{2}$ & $V_{5}$ & $\mathrm{~V}_{2}$ & $\mathrm{~V}_{5}$ \\
\hline Patient $1(6 \mathrm{CC})$ & 180 & 45.8 & 187 & 42.4 & 221 & 58 \\
\hline Patient 2(7 CC) & 162 & 49 & 180 & 47 & 282 & 52.6 \\
\hline Patient 3(7.12 CC) & 221 & 54.17 & 222 & 53.15 & 234 & 54.1 \\
\hline Patient 4(8.47 CC) & 225 & 61.2 & 239 & 55.7 & 248 & 64.3 \\
\hline Patient5(10.2 CC) & 263 & 67.1 & 271 & 63 & 294 & 72.7 \\
\hline Patient6(11.88CC) & 272 & 70 & 312 & 68 & 358 & 84 \\
\hline Patient7(12.12CC) & 361 & 74.3 & 402 & 72.3 & 450 & 90 \\
\hline Patient8(13.1 CC) & 326 & 77.5 & 472 & 76 & 504 & 107 \\
\hline Patient9(14.6 CC) & 354 & 90.9 & 418 & 84.8 & 468 & 121 \\
\hline Patient 10(15.7 CC) & 246 & 88.7 & 407 & 88 & 467 & 133 \\
\hline Mean $\pm S D(10.6 \pm 3.4 C C$ & $261 \pm 68.7$ & $67.9 \pm 15.5$ & $311 \pm 106$ & $65 \pm 15.5$ & $352.6 \pm 110.3$ & $83.7 \pm 28.7$ \\
\hline \multicolumn{7}{|c|}{ P Value (ANOVA) for com } \\
\hline \multicolumn{7}{|c|}{ P Value (ANOVA) } \\
\hline
\end{tabular}

Figure 8 represents the volume of the patient body that receives (a) 2 Gy and (b) 5 Gy.

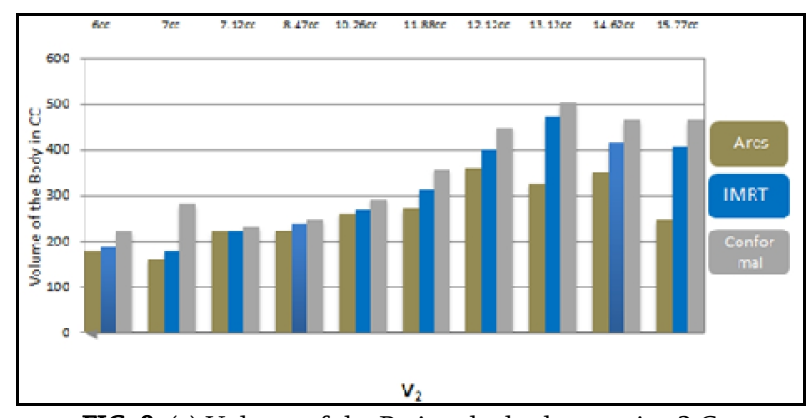

FIG. 8: (a) Volume of the Patient body that receive 2 Gy.

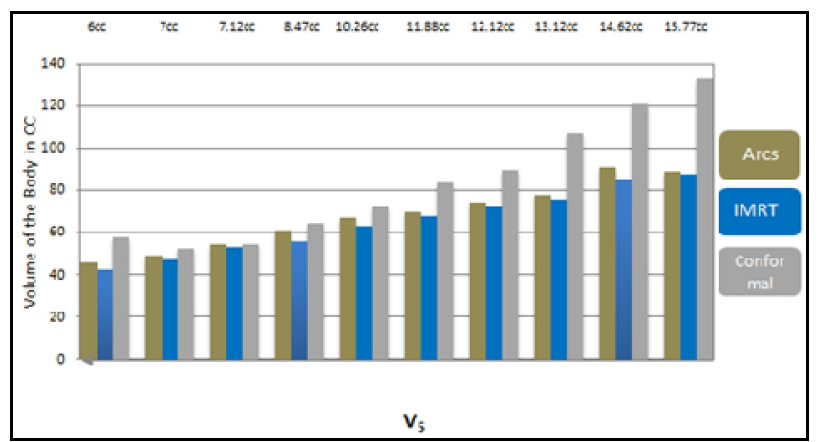

FIG. 8: (b) Volume of the Patient body that receive 5 Gy 
TABLE 5: Summarizes the Monitor Units (MUs) required for each techniques.

\begin{tabular}{cccc}
\hline \hline & \multicolumn{3}{c}{ MUs } \\
\cline { 2 - 4 } Patient 1(6 CC) & 1610 & IMRT & Conformal \\
Patient 2(7 CC) & 1618 & 3235 & 1713 \\
Patient 3(7.12 CC) & 1543 & 3341 & 1742 \\
Patient 4(8.47 CC) & 1588 & 3373 & 1784 \\
Patient 5(10.26 CC) & 1609 & 3390 & 1721 \\
Patient 6(11.88 CC) & 1596 & 3630 & 1743 \\
Patient 7(12.12 CC) & 1699 & 3719 & 1677 \\
Patient 8(13.12 CC) & 1715 & 3867 & 1709 \\
Patient 9(14.62 CC) & 1766 & 3865 & 1762 \\
Patient 10(15.77 CC) & 1794 & 3887 & 1829 \\
\hline Mean \pm SD(10.6 \pm 3.4 CC) & $1654 \pm 84$ & $3560 \pm 262$ & $1750 \pm 69$ \\
\hline P Value (ANOVA) for comparison between the three competing techniques: $<0.01$ \\
\multicolumn{5}{c}{ Pairwise comparison: } \\
& IMRT vs. Arcs: $<0.01$ \\
\hline \hline
\end{tabular}

\section{Estimation of treatment delivery time}

Recorded of the monitor units $(\mathrm{Mu})$ of each treatment plan gave us a reasonable data to estimate the treatment delivery time.

The results show that Arcs technique has the lowest volume of the body that received 2 Gy, IMRT is the next followed by 3DCRT and IMRT technique has the lowest volume of the body that received $5 \mathrm{~Gy}$, Arcs technique is the next followed by 3DCRT. The differences were not statistically significant $\mathrm{p}=0.126$ for $\mathrm{V} 2$ and $\mathrm{p}=0.118$ for $\mathrm{V} 5$, but these differences might be clinically significant that need more clinical discussion and investigation.

It is clear that the IMRT plan delivery time almost has two folded more than the two others techniques, and the arc the technique has the lowest estimated time compared to both IMRT and Conformal techniques. The differences were statistically significant $(P<0.01)$. All techniques have the same pre-treatment and quality assurance procedures time so the differences in the treatment delivery time among the competing three stereotactic techniques results in the differ MUs.

\section{Discussion}

The goal of SRS planning is to deliver the maximum dose to the tumor and the minimum dose to the surrounding healthy tissues. In this study 3 plans were generated Arcs, non-coplanar 3DCRT fixed field and non-coplanar IMRT. First Arc plan was made, Arc plan was made, optimized where the OARs were avoided as much as possible by selecting the optimal table positions, arc angles and the fitted cone size, then arc plan was converted into 3DCRT and IMRT with interval $45^{\circ}$ to have the same orientation and arrangement as the arc plan. To facilitate direct comparison between the competing techniques, the primary planning object was to cover $95 \%$ of the volume of PTV by $95 \%$ of the prescribed dose (1200 cGy).

In this study, the minimum dose for PTV was $93 \% \pm 1.5 \%$, $93.2 \% \pm 0.7 \%$ and $92.5 \% \pm 1.6$, the maximum dose was $104.3 \pm$ 2.6, $101.7 \pm 1.4$ and $106.1 \pm 2.6$, the dose inhomogeneity (the difference between the max and min dose) was $11.2 \% \pm 2.5 \%$, $8.6 \% \pm 1.5 \%$ and $13 \% \pm 2.1 \%$ for Arcs, IMRT and 3DCRT respectively (Table 1 ).

Conformity has been described using both the PITV ratio (Equation 1) and the CN (Equation 2). The PITV was $1.4 \pm$ $0.13,1.2 \pm 0.09$ and $1.4 \pm 0.09$ and the $\mathrm{CN}$ was $0.7 \pm 0.05,0.83$ \pm 0.05 and $0.67 \pm 0.04$ for Arcs, IMRT and 3DCRT respectively. As clear that the IMRT technique shows the highest dose conformity, Arcs the next homogeneity followed by 3DCRT. The differences were statistically significant among the three different techniques $(\mathrm{P}<0.01)$ for PITV and CN. For pairwise comparisons regarding PITV the difference were significant $(\mathrm{P}<0.003)$ for IMRT vs. Arcs and $(\mathrm{P}<0.01)$ for IMRT vs. 3DCRT and the pairwise comparisons were not statistically significant $(\mathrm{P}=0.598)$ for Arcs vs 3DCRT. The pairwise comparisons regarding $\mathrm{CN}$ the difference were significant $(\mathrm{P}<0.01)$ for IMRT vs. Arcs and $(\mathrm{P}<0.01)$ for IMRT vs. 3DCRT and the pairwise comparisons were not statistically significant $(P=0.405)$ for Arcs vs. 3DCRT

The PITV recommended in the Radiation Therapy Oncology Group (RTOG) radiosurgery guidelines is probably the most frequently quoted. A conformity index equal to 1 corresponds to ideal conformation. A conformity index greater than 1 indicates that the irradiated volume is greater than the target volume and includes healthy tissues. If the conformity index is less than 1 , the target volume is only partially irradiated. According to RTOG guidelines, ranges of conformity index values have been defined to determine the 
quality of conformation, because a value of 1 is rarely obtained. If the conformity index is situated between 1 and 2, treatment is considered to comply with the treatment plan; an index between 2 and 2.5, or 0.9 and 1, is considered to be a minor violation, and an index less than 0.9 or more than 2.5 is considered to be a major violation. However, this index presents a major drawback. ${ }^{15}$ It can never take into account the degree of spatial intersection of two volumes or their shapes. In extreme cases, it may be equal to 1 while these two volumes are situated away from each other and present entirely different shapes Figure 9 and the PITV not taken the healthy tissue into account.

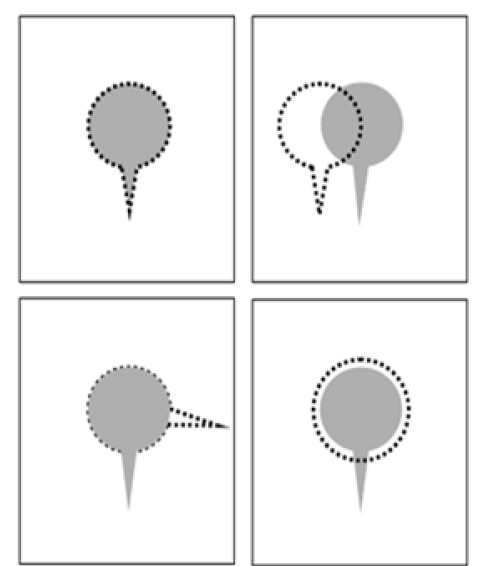

FIG. 9: Four possibilities for which the PITV ratio is equal to 1 (target volume, shaded; volume of reference isodose, enclosed in black dashes) Figure was taken from Feuvret et al..$^{15}$

To compensate for the defects of The PITV and To improve treatment evaluation van't Riet et al. ${ }^{8}$ proposed The CN which take into account all volume parameters of the lesion and healthy tissues. In our study we faced a major problem in the calculation of the $\mathrm{CN}$ because the software does not support all the parameters required to calculate the CN. This problem has been overcome manually by calculation of the $\mathrm{V}_{\mathrm{RI}}$ from help structure delineated over all the volume of the body. As example to calculate $\mathrm{V}_{\mathrm{RI}}$ (assume 95\% isodose line is the reference isodose) represented in Figure 10.a and from DVH Figure 10.b. The intersection of the help structure with 95\% dose represents the VRI as illustrated in Figure 10.
In this study IMRT techniques shows superiority in Dose Homogeneity, Conformity Index, Conformal Number and has the lowest integral dose followed by Arcs and 3DCRT techniques that might be results in defining field shapes by segments minimize the irradiating volume where the dose optimization in IMRT don't need to add a margin around the target volume and the beam boundary margin is being automatically minimized for IMRT inverse the 3DCRT which need to add 3-4 mm margin around the PTV to achieve the target coverage criteria this increase the normal tissue irradiation and the integral dose, and the disadvantage of Arcs technique with the circular cones is that cones can't fitted conformaly some of irregular shapes that result in increasing the dose outside the PTV Figure 10. The minimizing the mean field sizes give the IMRT technique the superiority in the dose fall-off outside the PTV. Table 6 shows the mean field sizes for the three competing techniques.

As shown in Table 6 the mean of equivalent square field sizes were $19.26 \pm 2.3,17.62 \pm 2.31$ and $20.44 \pm 2.50$ for Arcs, IMRT and 3DCRT respectively. It is clear that IMRT helped to reduce the mean equivalent field sizes by $9 \%$ and 3DCRT increase the mean equivalent field sizes by $6 \%$ compared to Arcs technique. The field sizes variation illustrate why IMRT has the lowest integral dose $(2.31 \pm 0.37 \mathrm{~J})$ followed by Arcs $(2.44 \pm 0.36 \mathrm{~J})$ and 3DCRT $(2.6 \pm 0.37 \mathrm{~J})$.

Field shaping by cones has some drawbacks that was specific for irregular shapes where the selection of the most fitted cone field size related to the biggest dimensions in the irregular shape, so we found despite of some targets have different sizes but shaping by the same cone field size, this clear in Table 6 for patient 7, patient 9, Patient 10 have different field sizes (12.12, 14.62, 15.77 respectively) and the same mean equivalent square field sizes (Figure 11) this might be result in the most fitted cone size designed to conform the longest distance between the points of the circumference of the target in the 3 dimensions, in contrast for IMRT and 3DCRT has been observed that the mean equivalent field size proportional with the size of the target as shown in Table 6 , this result in the mMLC designed to conform the target as a volume in the 3 dimensions, this deduction give privilege for using mMLC over using the Arcs technique.
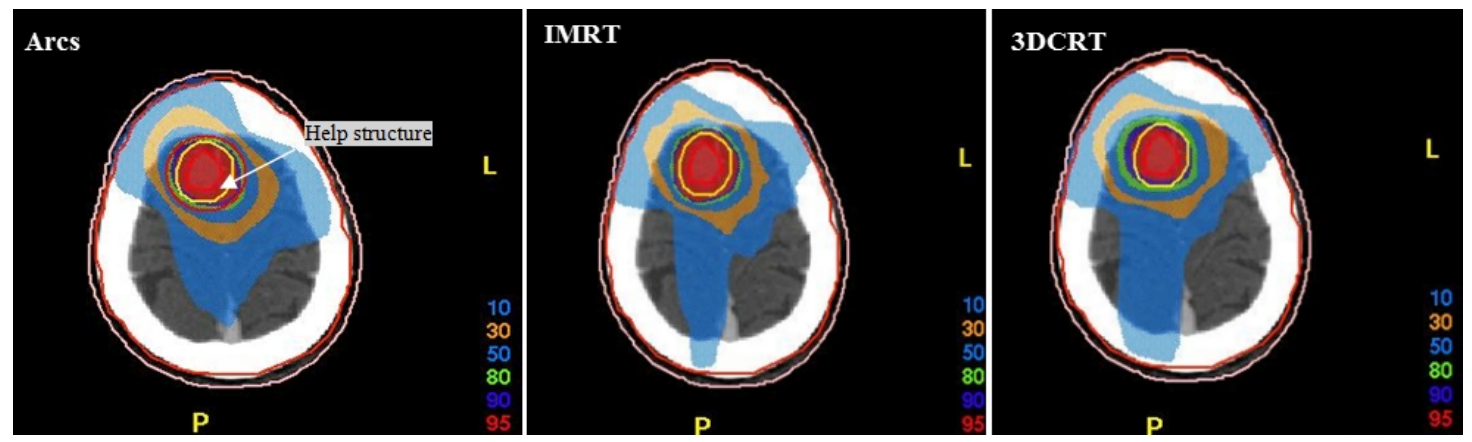

FIG. 10: (a) An example for relative isodose lines for the three different techniques. 


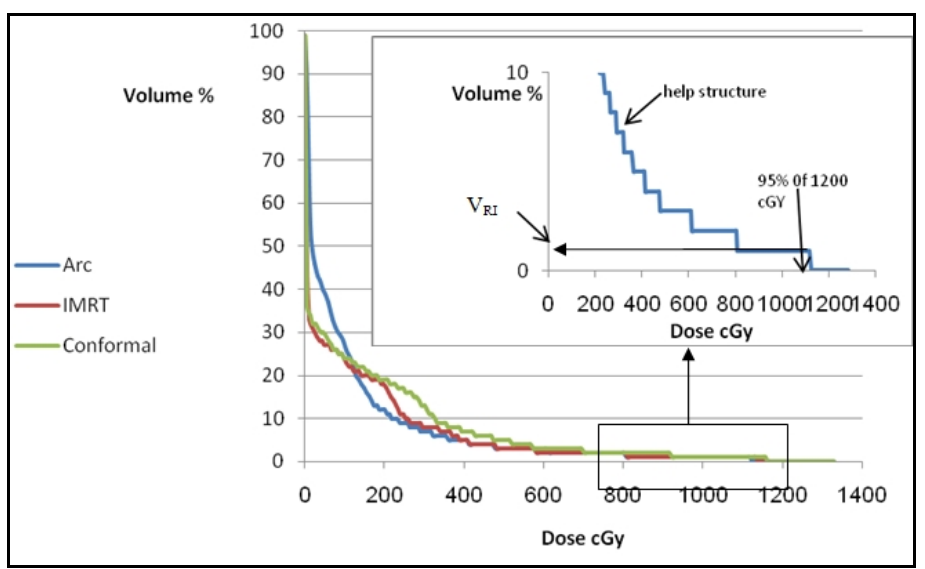

FIG. 10: (b) Extraction the VRI using the DVH of the help structure. Graph illustrates the method of calculation of the VRI.

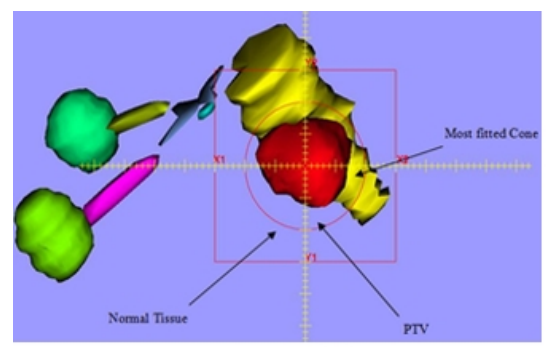

Arcs Technique

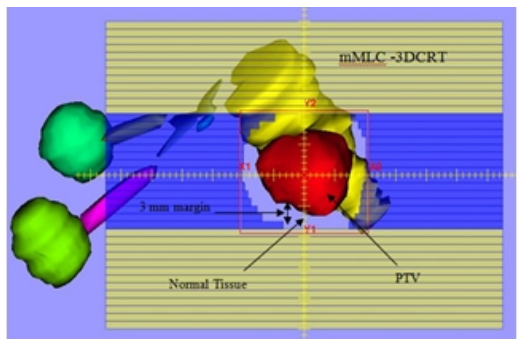

3DCRT Technique

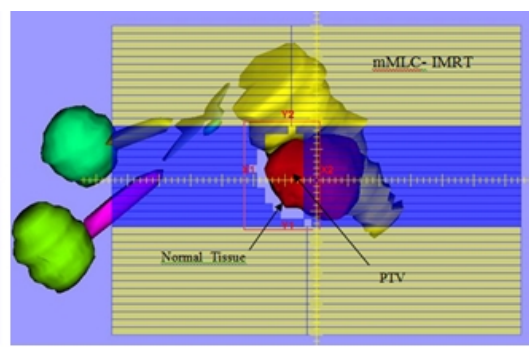

IMRT Technique

FIG. 10: An example of field size shaping for the three competing techniques:(a) Arcs,(b) 3DCRT, (c) IMRT techniques.

TABLE 6: Listed the mean of equivalent square field sizes of the three competing techniques for all patients.

\begin{tabular}{lccc}
\hline \hline & \multicolumn{3}{c}{ The mean of equivalent square field sizes $\left(\mathrm{cm}^{2}\right)$} \\
\cline { 2 - 4 } & Arcs & IMRT & Conformal \\
\cline { 2 - 4 } Patient 1(6 CC) & 14.5 & 14.04 & 17.12 \\
Patient 2(7 CC) & 17.4 & 15.2 & 18.16 \\
Patient 3(7.12 CC) & 17.4 & 15.2 & 18.23 \\
Patient 4(8.47 CC) & 18.72 & 17.06 & 18.87 \\
Patient 5(10.26 CC) & 19.5 & 17.58 & 19.8 \\
Patient 6(11.88 CC) & 20.04 & 17.94 & 20.44 \\
Patient 7(12.12 CC) & 21.36 & 18.3 & 21.64 \\
Patient 8(13.12 CC) & 20.97 & 19.53 & 21.74 \\
Patient 9(14.62 CC) & 21.36 & 20.37 & 23.64 \\
Patien10(15.77 CC) & 21.36 & 21.02 & 24.8 \\
\hline Mean $\pm S D(10.6 \pm 3.4$ CC) & $19.26 \pm 2.3$ & $17.62 \pm 2.31$ & $20.44 \pm 2.50$ \\
\hline \hline
\end{tabular}

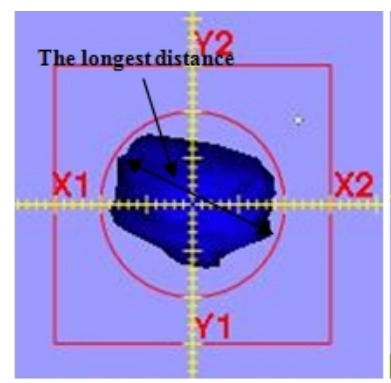

(a)

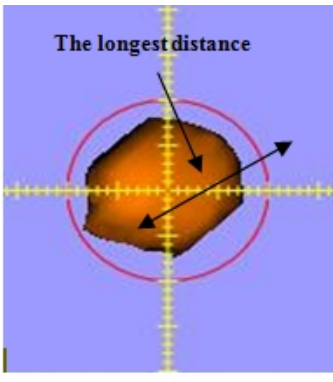

(b)

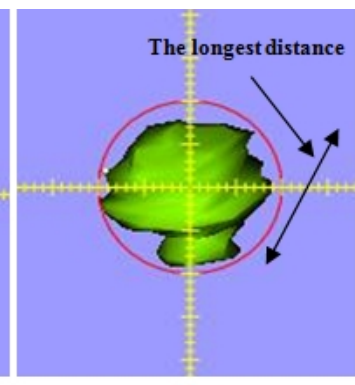

(c)

FIG. 11: Beam Eye View (BEV) shows the same cone size $(4 \mathrm{~cm})$ for different shape size a) $12.12 \mathrm{cc}, \mathrm{b}) 14.62 \mathrm{cc}, \mathrm{c}) 15.77 \mathrm{cc}$.

The volumes in $\mathrm{cm}^{3}$ that receive $2 \mathrm{~Gy}$ and $5 \mathrm{~Gy}$ have been recorded Table 4.4., the results shown that the Arcs technique has the lowest volume receiving $2 \mathrm{~Gy}$, followed by
IMRT and 3DCRT has the highest, in addition the volume receiving 5 Gy was the lowest in IMRT followed by Arcs and 3DCRT has the highest. This mean that Arcs technique re- 
duce the dose away the target rather than near the target this might result in for point sources as LINAC based SRS, the geometric penumbra is inversely proportional to the source to diaphragm distance, the geometric penumbra is reduced which helps increase the dose fall off. In contrast with mMLC attached to the head of linac, the geometric penumbra of mMLC is larger, so cones have dose sharp fall-off more than the mMLC but the increasing the equivalent field sizes in Arcs compared to IMRT cause the increasing dose receiving 5Gy around the target for Arcs technique. Based on these results, we can deduce that the influence of increasing field sizes for Arcs more than the influence of increasing of MUs and leaf leakage for IMRT for the dose near the target(V5) less for the dose away the target(V2).

Only a few studies comparing Arcs, IMRT, 3DCRT stereotactic techniques have been published. Kubo et al. ${ }^{12}$ performed a planning study involved 11 patients they found that for non-spherical targets treatment planning is relatively intuitive with mMLC-based radiosurgery. Moreover, a lower dose of radiation is delivered to normal tissue with mMLC-based radiosurgery than arc-based radiosurgery, the authors conclusion is similar to ours in the general idea. However we expressed the low doses by V2 and V5 because some models of radiation carcinogenesis suggest that the dose-response relationship is linear up to until a dose $6 \mathrm{~Gy}$. Cardinale et al. ${ }^{13}$ compared the dose conformity and normal brain dose characteristics of three different stereotactic techniques (arcs, non-coplanar fixed fields and intensity modulated) for various nonspherical target shapes of 3 intracranial test targets. The present study conformed the conclusion that IMRT has the superiority in dose conformity for hemisphere and irregular targets, but they didn't define what are the high and low dose as Kuboet al. ${ }^{12}$ where we defined it to be V2 that more favorable with Arcs technique and V5 that more favorable with IMRT.

The potential of single-fraction IMRT SRS has not yet been fully released. Ernst-Stecken et al. ${ }^{14}$ evaluated radiosurgery intensity-modulated stereotactic radiosurgery (IMRT-SRS) and dynamic conformal arc (DCA) techniques in small skull-base tumors. They concluded that RTOG radiosurgery guidelines were best met by the DCA rather than IMSRS approach for the treatment of small skull-base lesions. The IMRT-SRS approach will increase the time for planning, dose delivery and integral dose to the brain. Furthermore, the results presented in this study were based on dose calculations performed in Radionics treatment planning system $\mathrm{X}$-Knife radionics. Future studies may include the use of different treatment planning systems and dose calculation algorithms such as collapsed cone convolution superposition algorithm ${ }^{16}$ and Acuros XB algorithm ${ }^{17}$, and how these algorithms affect the peripheral dose and conformity index.

\section{Conclusion}

The conformity index, dose homogeneity and the outfield dose are important aspects of plan quality, although they do not always receive clinically attention. Our results have been showed that the superiority of the IMRT in conformity, dose homogeneity and the lowest volume that received 5 Gy, Arcs technique has the superiority in lower treatment delivery time than IMRT and the lowest volume that received $2 \mathrm{~Gy}$. The 3DCRT don't present a significant advantage among the competing techniques. Oncologist should be alert of the possibility of significantly increasing the secondary cancer risk particularly for pediatric patients because children have a greater risk of developing a secondary cancer due to their tissue's higher radiation sensitivity and their longer survival times.

\section{Conflict of interest}

The authors declare that they have no conflicts of interest. The authors alone are responsible for the content and writing of the paper.

\section{References}

1. Schell MC, Larson DA, Leavitt DD, et al. Stereotactic Radiosurgery. American Association of Physicists in Medicine (AAPM) 1995.

2. Taylor ML, McDermott LN, Johnston PN, et al. Stereotactic fields shaped with a micro-multileaf collimator: systematic characterization of peripheral dose. Phys Med Biol 2010; 55:873-81.

3. Majali M, Novotny J, Novotny J Jr. Measurement of the peripheral doses for linac stereotactic radiotherapy. Radiat Prot Dosimetry 2003; 106:247-52.

4. $\mathrm{Wu} \mathrm{QR}, \mathrm{Wessels} \mathrm{BW,} \mathrm{Einstein} \mathrm{DB,} \mathrm{et} \mathrm{al.} \mathrm{Quality} \mathrm{of}$ coverage: conformity measures for stereotactic radiosurgery. J Appl Clin Med Phys 2003; 4:374-81.

5. Taylor ML, Kron T, Franich RD. Assessment of out-of-field doses in radiotherapy of brain lesions in children. Int J Radiat Oncol Biol Phys 2011; 79:927-33.

6. Di Betta E, Fariselli L, Bergantin A, et al. Evaluation of the peripheral dose in stereotactic radiotherapy and radiosurgery treatments. Med Phys 2010; 37:3587-94.

7. Sulieman A, Theodorou K, Kappas C. Entrance and peripheral doses measurements during radiotherapy. Journal of Science and Technology 2011; 12:20-28.

8. van't Riet A, Mak AC, Moerland MA, et al. A conformation number to quantify the degree of conformality in brachytherapy and external beam irradiation: application to the prostate. Int $J$ Radiat Oncol BiolPhys 1997; 37:731-6. 
9. Shaw E, Kline R, Gillin M, et al. Radiation Therapy Oncology Group: radiosurgery quality assurance guidelines. Int J Radiat Oncol Biol Phys 1993; 27:1231-9.

10. Oliver M, Ansbacher W, Beckham WA. Comparing planning time, delivery time and plan quality for IMRT, RapidArc and Tomotherapy. J Appl Clin Med Phys 2009; 10:3068.

11. Ruben JD, Davis S, Evans C, et al. The effect of intensity-modulated radiotherapy on radiation-induced second malignancies. Int $J$ Radiat Oncol Biol Phys 2008; 70:1530-6.

12. Kubo HD, Pappas CT, Wilder RB. A comparison of arc-based and static mini-multileaf collimator-based radiosurgery treatment plans. Radiother Oncol 1997; 45:89-93.

13. Cardinale RM, Benedict SH, Wu Q et al. A comparison of three stereotactic radiotherapy techniques; ARCS vs. noncoplanar fixed fields vs. in- tensity modulation. Int $J$ Radiat Oncol Biol Phys 1998; 42:431-6.

14. Ernst-Stecken A, Lambrecht U, Ganslandt O, Mueller R, Fahlbusch R, Sauer R,Grabenbauer G. Radiosurgery of small skull-base lesions. No advantage for intensity-modulated stereotactic radiosurgery versus conformal arc technique. Strahlenther Onkol 2005; 181:336-44.

15. Feuvret L, Noel G, Mazeron JJ, Bey P. Conformity index: a review. Int J Radiat Oncol Biol Phys 2006; 64:333-42.

16. Oyewale S. Dose prediction accuracy of collapsed cone convolution superposition algorithm in a multi-layer inhomogenous phantom. Int J Cancer Ther Oncol 2013; 1:01016.

17. Rana S. Clinical dosimetric impact of Acuros XB and analytical anisotropic algorithm (AAA) on real lung cancer treatment plans: review. Int J Cancer Ther Oncol 2014; 2:02019. 